VAND-TH-01-1

hep-ph/0101091

January 9, 2001

\title{
Absolute Neutrino Mass Determination
}

\author{
H. Päs * and T.J. Weiler ${ }^{\dagger}$ \\ Department of Physics and Astronomy \\ Vanderbilt University \\ Nashville, TN 37235, USA
}

\begin{abstract}
We discuss four approaches to the determination of absolute neutrino mass. These are the measurement of the zero-neutrino double beta decay rate, of the tritium decay end-point spectrum, of the cosmic ray spectrum above the GZK cutoff (in the Z-burst model), and the cosmological measurement of the power spectrum governing the CMB and large scale structure. The first two approaches are sensitive to the mass eigenstates coupling to the electron neutrino, whereas the latter two are sensitive to the heavy component of the cosmic neutrino background. All mass eigenstates are related by the $\delta m^{2}$ 's inferred from neutrino oscillation data. Consequently, the potential for absolute mass determination of each of the four approaches is correlated with the other three, in ways that we point out.
\end{abstract}

PACS: 14.60.Pq, 23.40.Bw, 96.40.Tv, 98.80.Es.

*E-mail: heinrich.paes@vanderbilt.edu

†E-mail: tom.weiler@vanderbilt.edu 


\section{INTRODUCTION}

An ongoing experimental effort of high importance is the determination of the neutrino mass eigenvalues. The absolute scale of neutrino masses, a crucial datum for reconstructing physics beyond the Standard Model, is unknown. Presently, upper bounds on the absolute neutrino mass are provided by the tritium decay end-point spectral measurement, by cosmology, and by zero-neutrino double beta decay $(0 \nu \beta \beta)$.

The present tritium decay upper bound on each [1] of the three neutrino mass eigenstates is $2.3 \mathrm{eV}$ (95\% C.L.) [2]. An upper bound from cosmological structure formation is more stringent but also more model-dependent. For three degenerate neutrino masses, the constraint on the individual neutrino mass eigenstates is $m_{j}<1.8 \mathrm{eV}$ for large $\Omega_{m}$, and $m_{j}<0.6 \mathrm{eV}$ for $\Omega_{m} \sim 0.3$ [3]; $\Omega_{m}$ is the matter fraction of the closure density. The present $0 \nu \beta \beta$ upper limit on the ee element $m_{e e}$ of the flavor-basis Majorana neutrino mass matrix is $0.27 \mathrm{eV}$ 幽. Fortunately for $0 \nu \beta \beta$ searches, models which generate small neutrino masses overwhelmingly favor Majorana neutrinos over Dirac neutrinos [5] (but see [6] for a small Dirac masses generated by brane-bulk interactions).

To determine an absolute neutrino mass below $1 \mathrm{eV}$ is a true experimental challenge. The three approaches just mentioned have the potential to accomplish the task. Anticipated improvements in these approaches are

(i) larger versions of the tritium end-point distortion measurements;

(ii) the comparison of more-precisely determined early-Universe temperature perturbations (MAP [7] and PLANCK [8] experiments) to the present-day large-scale structure distributions of matter (to be measured by SDSS [9] and 2dF [10]); and

(iii) larger $0 \nu \beta \beta$ experiments (GENIUS and EXO are proposed).

In addition there is a fourth possibility:

(iv) the extreme-energy cosmic-ray experiments (AGASA [11, HiRes [12], Auger [13], Telescope Array [14, EUSO/OWL [15]) in the context of the recently emphasized Z-burst model 16, 17 .

Still another approach to neutrino mass determination, measuring the arrival-time profile of neutrinos from supernovae, seems not quite capable of breaking the sub-eV barrier [18].

The Z-burst and cosmic structure measurements are sensitive to the heavier neutrino 
masses (and the cosmological neutrino background), while the tritium and $0 \nu \beta \beta$ experiments are sensitive to different linear combinations of whichever masses are coupled to $\nu_{e}$. Neutrino oscillation interpretations of solar, atmospheric, and LSND data produce nonzero values for neutrino mass-squared differences, and so relate all neutrino masses. Accordingly, the expectations of the four approaches listed above to absolute neutrino mass determination are related. Any positive finding in one approach requires concordance in the other three. It is the purpose of this work to reveal the relations among the reaches of the four approaches.

We begin with a discussion of the neutrino mass-relations inferred from oscillation interpretations of recent data. We will conservatively consider a three-neutrino Universe, omitting the uncorroborated data from the LSND experiment; in the future, the miniBooNE experiment at Fermilab will rule on the validity of the LSND measurement. Specifically, we label the mass eigenstates as $m_{3}>m_{2}>m_{1}$, and denote the mass-squared differences as

$$
m_{3}^{2}=m_{1}^{2}+\delta m_{\mathrm{atm}}^{2}, \quad \text { and } \quad m_{2}^{2}=m_{1}^{2}+\delta m_{\mathrm{sun}}^{2}
$$

with $\delta m_{\text {atm }}^{2}$ and $\delta m_{\text {sun }}^{2}$ positive. Oscillations are directly sensitive to these nonzero neutrino mass-squared differences. The alternative splitting ("inverted hierarchy") with two heavy states and a single light state is discussed briefly in the conclusions section; it is disfavored according to a recent analysis [19] of the neutrino spectrum from SN1987A, unless the mixing element $U_{e 1}$ is large.

The solar and atmospheric neutrino oscillation interpretations, and the nonobservation of short-baseline $\nu_{e}$ disappearance in the CHOOZ experiment, provide valuable information on the $\delta m^{2}$ 's and mixing angles. The most recent global data-analysis in a three neutrino framework yields the following favored regions [20]:1]

- Solar neutrino data favor $\nu_{e}-\nu_{\ell}$ oscillations within the large mixing-angle (LMA) MSW solution: $\delta m_{\mathrm{sun}}^{2}=3 \times 10^{-5} \mathrm{eV}^{2}$, with a $90 \%$ C.L. of $(1-10) \times 10^{-5} \mathrm{eV}$; $\tan ^{2} \theta_{\text {sun }}=0.5$, with a $90 \%$ C.L. of $(0.2-0.6)$.

\footnotetext{
${ }^{1}$ It has become customary to express a mixing angle sensitive to matter effects, such as the solar angle, as $\tan \theta$ rather than $\sin 2 \theta$ to account for the octant of the "dark side", $\pi / 4<\theta \leq \pi / 2$.
} 
Also allowed at $90 \%$ C.L. is a small region in the

LOW-QVO (quasi-vacuum oscillation) regime:

$\delta m_{\text {sun }}^{2}=10^{-7} \mathrm{eV}^{2}$,

$\tan ^{2} \theta_{\text {sun }}=(0.6-0.8)$.

The small mixing-angle (SMA) MSW solution at

$\delta m_{\text {sun }}^{2}=(4-9) \cdot 10^{-5} \mathrm{eV}^{2}$,

$\tan ^{2} \theta_{\text {sun }}=(0.2-1) \cdot 10^{-3}$

is disfavored at $90 \%$ C.L. but viable at $95 \%$ C.L.

- Atmospheric neutrino data are explained by maximal $\nu_{\mu}-\nu_{\tau}$ oscillations with: $\delta m_{\mathrm{atm}}^{2}=3 \times 10^{-3} \mathrm{eV}^{2}$, and a $90 \%$ C.L. of $(1.6-5) \times 10^{-3} \mathrm{eV}^{2}$;

$\sin ^{2} 2 \theta_{\text {atm }}>0.85$.

It should be stressed also that at larger C.L. the large-angle solution for solar neutrinos can extend over nearly the entire region from $\delta m_{\text {sun }}^{2}=10^{-10} \mathrm{eV}^{2}$ up to $\delta m_{\text {sun }}^{2}=10^{-3} \mathrm{eV}^{2}$. Also, data from Supernova1987A have recently been re-analyzed in the context of the various solar neutrino solutions. The result is that the LOW-QVO solutions are disfavored at $4 \sigma$ compared to the SMA and LMA solutions [21] over most of the supernova parameter space.

The mass-squared differences inferred from solar and atmospheric measurements imply lower bounds on the masses $m_{3}$ and $m_{2}$. The atmospheric bound is $m_{3} \geq \sqrt{\delta m_{\mathrm{atm}}^{2}} \sim 0.05 \mathrm{eV}$, which is encouraging for mass-sensitive experiments. The relation among the three masses enforced by the oscillation interpretation of solar and atmospheric data is plotted is Fig. 1. Also shown in the figure are the present tritium and cosmological upper bounds on absolute neutrino mass. The mass-squared differences inferred from data show a definite hierarchy: $\delta m_{\text {sun }}^{2} \ll \delta m_{\text {atm }}^{2}$ by probably a factor of 30 or more. As seen in Fig. 1, this may or may not imply a mass hierarchy. If $m_{1} \gg \sqrt{\delta m_{\mathrm{atm}}^{2}} \sim 0.05 \mathrm{eV}$, then all three neutrino masses are nearly degenerate, while if $m_{1} \lesssim \sqrt{\delta m_{\text {atm }}^{2}} \sim 0.05 \mathrm{eV}$, then the three masses are not degenerate. The degenerate possibility has been preferred in cold+hot darkmatter models to account for observed large-scale structures. However, the need for the hot component is mitigated by the cosmological constant introduced to explain high red-shift 
Type Ia supernovae observations.

If $m_{1} \gg \sqrt{\delta m_{\mathrm{sun}}^{2}} \sim 0.003 \mathrm{eV}$, then the two lightest neutrino masses $m_{1}$ and $m_{2}$ are nearly degenerate. With the exception of a futuristic 10 ton version of GENIUS, the reach of the four approaches considered in this work does not extend down to as low as $0.003 \mathrm{eV}$. Accordingly, in what follows we take $m_{1}$ and $m_{2}$ to be degenerate.

We return to the four approaches to absolute neutrino-mass determination. Because the relevance of extreme-energy cosmic rays (EECRs) to neutrino mass determination via the Z-burst model is the least known of the approaches, and because data already exist which in the context of the model implicate an absolute neutrino mass (in the range 0.1 to $1.0 \mathrm{eV}$ ), we consider the Z-burst approach first. The model is speculative. However, if it is validated as the explanation of EECR puzzles, the payoff is big. Not only is the absolute mass of the neutrino revealed, but also the existence of the cosmic neutrino background (CNB) liberated one second after the Big Bang.

\section{THE Z-BURST MODEL FOR EECR'S}

It was expected that the EECR primaries would be protons from outside the galaxy, produced in Nature's most extreme environments such as the tori or radio hot spots of active galactic nuclei (AGN). Indeed, cosmic ray data show a spectral flattening just below $10^{19} \mathrm{eV}$ which can be interpreted as a new extragalactic component overtaking the lower energy galactic component; the energy of the break correlates well with the onset of a Larmor radius for protons too large to be contained by the Galactic magnetic field. It was further expected that the extragalactic spectrum would reveal an end at the Greisen-KuzminZatsepin $(\mathrm{GZK})$ cutoff energy of $E_{\mathrm{GZK}} \sim 5 \times 10^{19} \mathrm{eV}$. The origin of the GZK cutoff is the degradation of nucleon energy by the resonant scattering process $N+\gamma_{2.7 K} \rightarrow \Delta^{*} \rightarrow N+\pi$ when the nucleon is above the resonant threshold $E_{\mathrm{GZK}}$ for $\Delta^{*}$ production. The concomitant energy-loss factor is $\sim(0.8)^{D / 6 \mathrm{Mpc}}$ for a nucleon traversing a distance $D$. Since no AGN-like sources are known to exist within $100 \mathrm{Mpc}$ of earth, the energy requirement for a proton arriving at earth with a super-GZK energy is unrealistically high. Nevertheless, to date more than twenty events with energies at and above $10^{20} \mathrm{eV}$ have been observed [22].

The spectral break just below $\sim 10^{19} \mathrm{eV}$ and the super-GZK events from the AGASA 
experiment are displayed in Fig. 2.

Several solutions have been proposed for the origin of these EECRs, ranging from unseen Zevatron accelerators $\left(1 \mathrm{ZeV}=10^{21} \mathrm{eV}\right)$ and decaying supermassive particles and topological defects in the Galactic vicinity, to exotic primaries, exotic new interactions, and even exotic breakdown of conventional physical laws [23]. A rather conservative and economical scenario involves cosmic ray neutrinos scattering resonantly on the cosmic neutrino background (CNB) predicted by Standard Cosmology, to produce Z-bosons [17]. These Z-bosons in turn decay to produce a highly boosted "Z-burst", containing on average twenty photons and two nucleons above $E_{\mathrm{GZK}}$ (see Fig. 3). The photons and nucleons from Z-bursts produced within 50 to $100 \mathrm{Mpc}$ of earth can reach earth with enough energy to initiate the air-showers observed at $\sim 10^{20} \mathrm{eV}$.

The energy of the neutrino annihilating at the peak of the Z-pole is

$$
E_{\nu_{j}}^{R}=\frac{M_{Z}^{2}}{2 m_{j}}=4\left(e V / m_{j}\right) \mathrm{ZeV} .
$$

The resonant-energy width is narrow, reflecting the narrow width of the Z-boson: at FWHM $\Delta E_{R} / E_{R} \sim \Gamma_{Z} / M_{Z}=3 \%$. The mean energies of the $\sim 2$ baryons and $\sim 20$ photons produced in the $\mathrm{Z}$ decay are easily estimated. Distributing the Z-burst energy among the mean multiplicity of 30 secondaries in Z-decay [24], one has

$$
\left\langle E_{p}\right\rangle \sim \frac{E_{R}}{30} \sim 1.3\left(\frac{\mathrm{eV}}{m_{j}}\right) \times 10^{20} \mathrm{eV} .
$$

The photon energy is further reduced by an additional factor of 2 to account for their origin in two-body $\pi^{0}$ decay:

$$
\left\langle E_{\gamma}\right\rangle \sim \frac{E_{R}}{60} \sim 0.7\left(\frac{\mathrm{eV}}{m_{j}}\right) \times 10^{20} \mathrm{eV}
$$

Even allowing for energy fluctuations about mean values, it is clear that in the Z-burst model the relevant neutrino mass cannot exceed $\sim 1 \mathrm{eV}$. On the other hand, the neutrino mass cannot be too light of the predicted primary energies will exceed the observed event energies.2 In this way, one obtains a rough lower limit on the neutrino mass of $\sim 0.1 \mathrm{eV}$ for

\footnotetext{
${ }^{2}$ Also, the neutrino mass cannot be too small without pushing the primary neutrino flux to unattractively higher energies.
} 
the Z-burst model, when allowance is made for an order of magnitude energy-loss for those secondaries traversing 50 to $100 \mathrm{Mpc}$.

The necessary conditions for the viability of this model are a sufficient flux of neutrinos at $\gtrsim 10^{21} \mathrm{eV}$ and a neutrino mass scale of the order $0.1-1 \mathrm{eV}$ [16, 17]. The first condition seems challenging [25], while the second is quite natural in view of the recent oscillation data (see Fig. 1).

It is worth remarking that the cosmic fluxes of the three neutrino mass-eigenstates are virtually guaranteed to be nearly equal as a result of the $\nu_{\mu}-\nu_{\tau}$ near-maximal mixing observed in atmospheric data. This comes about as follows: For extragalactic neutrinos produced in $\pi^{ \pm}$decay, the original flavor ratio $\nu_{e}: \nu_{\mu}: \nu_{\tau} \sim 1: 2: 0$ oscillates to $\sim 1: 1: 1$; for more exotic neutrino production from, e.g., string cusps, a flavor-neutral ratio of $1: 1: 1$ may be expected at the source. For both cases, an equal population of flavor states results for the cosmic flux. It then follows from unitarity of the mixing matrix that there is also an equal population of mass states in the flux. An equal population of mass states is also expected among the thermally produced CNB. The equal population of mass states in flux and CNB has interesting consequences. It follows that the relative Z-burst rate at each of the three resonant energies is given by the total neutrino flux value $F_{\nu}\left(E^{R}\right)$ at each resonant energy. If the neutrinos are mass degenerate, then a further consequence is that the Z-burst rate at $E_{R}$ is three times what it would be without degeneracy. This ameliorates slightly the formidable flux requirement.

The viability of the Z-burst model is enhanced if the CNB neutrinos cluster in our matterrich vicinity of the universe. The main constraints on clustering are two-fold. For very large scales, the Universe is too young to have experienced significant infall of matter. For smaller scales, the Pauli blocking of identical neutrinos sets a limit on density enhancement. As a crude estimate of Pauli blocking, one may use the zero temperature Fermi gas as a model of the gravitationally bound neutrinos. Requiring that the Fermi momentum of the neutrinos

3 The mass basis is the more relevant basis for annihilation on the nonrelativistic relic neutrinos; it is also the more physical basis when it is realized that flavor states traveling cosmic distances (the flux) or existing for cosmic ages (the CNB) will have decohered into mass states. 
does not exceed mass times the virial velocity $\sigma \sim \sqrt{M G / L}$ within the cluster of mass $M$ and size $L$, one gets 26

$$
\frac{n_{\nu_{j}}}{54 \mathrm{~cm}^{-3}} \lesssim 10^{3}\left(\frac{m_{j}}{\mathrm{eV}}\right)^{3}\left(\frac{\sigma}{200 \mathrm{~km} / \mathrm{s}}\right)^{3} .
$$

The virial velocity within our Galactic halo is a couple hundred $\mathrm{km} / \mathrm{sec}$. Thus it appears that Pauli blocking allows significant clustering on the scale of our Galactic halo only if $m_{j} \gtrsim 0.5 \mathrm{eV}$. An indicator of the neutrino mass sufficient to allow a 100-fold increase in the Galactic halo density of the CNB is shown on Fig. 4.

For rich clusters of galaxies, the virial velocities are a thousand $\mathrm{km} / \mathrm{s}$ or more. Thus, Pauli blocking does not exclude significant clustering on scales of tens of Mpc for $m_{j} \gtrsim 0.1 \mathrm{eV}$. However, on these large scales, the infall of matter integrated to the present time is probably insufficient to effect significant clustering.

\section{TRITIUM DECAY END-POINT LIMITS}

In tritium decay, the larger the mass states comprising $\bar{\nu}_{e}$, the smaller is the Q-value of the decay. The manifestation of neutrino mass is a reduction of phase space for the produced electron at the high energy end of its spectrum. An expansion of the decay rate formula about $m_{\nu_{e}}$ leads to the end point sensitive factor

$$
m_{\nu_{e}}^{2} \equiv \sum_{j}\left|U_{e j}\right|^{2} m_{j}^{2}
$$

where the sum is over mass states which can kinematically alter the end-point spectrum. If the neutrino masses are nearly degenerate, then unitarity of $U$ leads immediately to a

bound on $\sqrt{m_{\nu_{e}}^{2}}=m_{3}$. The design of larger tritium decay experiments to reduce the present $2.3 \mathrm{eV} m_{\nu_{e}}$ bound are under discussion; direct mass limits as low as $0.4 \mathrm{eV}$, or even $0.2 \mathrm{eV}$, may be possible in this type of experiment.

\section{CMB/LSS COSMOLOGICAL LIMITS}

According to Big Bang cosmology, the masses of nonrelativistic neutrinos are related to the neutrino fraction of closure density by $\sum_{j} m_{j}=40 \Omega_{\nu} h_{65}^{2} \mathrm{eV}$, where $h_{65}$ is the present 
Hubble parameter in units of $65 \mathrm{~km} / \mathrm{s} / \mathrm{Mpc}$. As knowledge of large-scale structure (LSS) formation has evolved, so have the theoretically preferred values for the hot dark matter (HDM) component, $\Omega_{\nu}$. In fact, the values have declined. In the once popular HDM cosmology, one had $\Omega_{\nu} \sim 1$ and $m_{\nu} \sim 10 \mathrm{eV}$ for each of the mass-degenerate neutrinos. In the cold-hot CHDM cosmology, the cold matter was dominant and one had $\Omega_{\nu} \sim 0.3$ and $m_{\nu} \sim 4 \mathrm{eV}$ for each neutrino mass. In the currently favored $\Lambda \mathrm{DM}$ cosmology, there is scant room left for the neutrino component. An analysis relating the cosmic microwave background (CMB) temperature fluctuations and the present-day LSS provides the limit. The power spectrum of early-Universe density perturbations, fossilized in the observed CMB fluctuations at the recombination epoch $z_{r} \sim 1100$, is processed by gravitational instabilities. However, the free-streaming relativistic neutrinos suppress the growth of fluctuations on scales below the horizon (approximately the Hubble size $c / H(z)$ ) until they become nonrelativistic at $z \sim m_{j} / 3 T_{0} \sim 1000\left(m_{j} / \mathrm{eV}\right)$. The result of simulation is a neutrino component constrained as $\sum_{j} m_{j}<5.5 \mathrm{eV}$ for all values of $\Omega_{m}$; and $m_{j}<0.6(0.9) \mathrm{eV}$ for each of three degenerate neutrinos and for $\Omega_{m}=0.3(0.4)$, all at $95 \%$ C.L. [3].

The Sloan Digital Sky Survey (SDSS) should measure the power spectrum of the LSS to $\sim 1 \%$ accuracy. Combining this with the CMB measurements expected from the MAP satellite, one can infer neutrino mass down to [27]

$$
\sum m_{\nu} \simeq 0.33\left(\frac{\Omega_{m} h_{65}^{2}}{0.3} \frac{3}{\mathrm{~N}}\right)^{0.8} \mathrm{eV}
$$

Here $\mathrm{N}$ is the number of degenerate neutrinos. The effect of a single neutrino state on the CMB anisotropy in $\Lambda$ DM models has also been discussed [28]. A sensitivity for MAP to $2 \mathrm{eV}$ neutrinos with temperature data alone, and to $0.5 \mathrm{eV}$ with polarization data included is estimated; for the PLANCK satellite a sensitivity to $0.5 \mathrm{eV}$ with temperature data alone and to $0.25 \mathrm{eV}$ with polarization data included is claimed.

Some caution is warranted in the cosmological approach to neutrino mass, in that the many cosmological parameters may conspire in various combinations to yield nearly identical CMB and LSS data. An assortment of very detailed data may be needed to resolve the possible "cosmic ambiguities". 


\section{NEUTRINOLESS DOUBLE BETA DECAY}

Neutrinoless double beta decay $(0 \nu \beta \beta)$ proceeds via the nuclear reaction ${ }_{Z}^{A} X \rightarrow{ }_{Z+2}^{A} X+2 e^{-}$. The rate is a sensitive tool for the measurement of the absolute mass-scale for Majorana neutrinos [29]. The observable measured in the amplitude of $0 \nu \beta \beta$ decay is the ee element of the neutrino mass-matrix in the flavor basis. Expressed in terms of the mass eigenvalues and neutrino mixing-matrix elements, it is

$$
m_{e e}=\left|\sum_{i} U_{e i}^{2} m_{i}\right|
$$

A reach as low as $m_{e e} \sim 0.01 \mathrm{eV}$ seems possible with proposed double beta decay projects

such as the 1 ton version of GENIUS [30] and EXO [31]. This provides a substantial improvement over the current bound, $m_{e e}<0.27 \mathrm{eV}$. In the far future, another order of magnitude in reach is available to the 10 ton version of GENIUS, should it be funded and commissioned.

For masses in the interesting range $\gtrsim 0.01 \mathrm{eV}$, the two light mass eigenstates are nearly degenerate and so the approximation $m_{1}=m_{2}$ is justified. Furthermore, the restrictive CHOOZ bound [32], $\left|U_{e 3}\right|^{2}<0.025$ in the three neutrino model (for $\delta m_{\mathrm{atm}}^{2} \geq 10^{-3} \mathrm{eV}^{2}$ ), allows two further simplifications. The first is that the contribution of the third mass eigenstate is nearly decoupled from $m_{e e}$ and so $U_{e 3}^{2} m_{3}$ may be neglected in the $0 \nu \beta \beta$ formula. The second is that the two-neutrino mixing approximation is valid, i.e. $U_{e 1} \approx e^{i \phi_{1}} \cos \theta_{\text {sun }}$ and $U_{e 2} \approx e^{i \phi_{2}} \sin \theta_{\text {sun. }}$. We label by $\phi_{12}$ the relative phase between $U_{e 1}^{2} m_{1}$ and $U_{e 2}^{2} m_{2}$. Then, employing the above approximations, we arrive at a very simplified expression for $m_{e e}$ :

$$
m_{e e}^{2}=\left[1-\sin ^{2}\left(2 \theta_{\text {sun }}\right) \sin ^{2}\left(\frac{\phi_{12}}{2}\right)\right] m_{1}^{2} .
$$

The two CP-conserving values of $\phi_{12}$ are 0 and $\pi$. These same two values give maximal constructive and destructive interference of the two dominant terms in eq. (8), which leads to upper and lower bounds for the observable $m_{e e}$ in terms of a fixed value of $m_{1}$ :

$$
\cos \left(2 \theta_{\text {sun }}\right) m_{1} \leq m_{e e} \leq m_{1}, \text { for fixed } m_{1}
$$

The upper bound becomes an equality, $m_{e e}=m_{1}$, for any of the solar solutions if $\phi_{12}=0$, and for the small-angle SMA solution $\left(\cos \left(2 \theta_{\text {sun }}\right) \approx 1\right)$ with any $\phi_{12}$. The lower bound 
depends on Nature's value of the mixing angle in the LMA and LOW-QVO solutions.f A consequence of eq. (10) is that for a given measurement of $m_{e e}$, the corresponding inference of $m_{1}$ is uncertain over the range $\left[m_{e e}, m_{e e} \cos \left(2 \theta_{\text {sun }}\right)\right]$ due to the unknown phase difference $\phi_{12}$.

Knowing the value of $\theta_{\text {sun }}$ better will improve the estimate of the inherent uncertainty in $m_{1}$. For the LMA solar solution, the forthcoming Kamland experiment should reduce the error in the mixing angle $\sin ^{2} 2 \theta_{\text {sun }}$ to \pm 0.1 [33]. However, it is unlikely that the inherent uncertainty in $m_{1}$ can be reduced beyond $\left(\cos 2 \theta_{\text {sun }}\right)^{-1}$, since there is no known way to measure the Majorana phase difference $\phi_{12}$. Ultimately, the inferences made for $m_{e e}$ from a positive $0 \nu \beta \beta$ result will also depend on the uncertainty in the charged-current nuclear matrix element. Currently this uncertainty is a factor of 2 to 3 . We ignore it in what follows.

A quantitative discussion of the reach of $0 \nu \beta \beta$ is presented in the next section.

\section{CORRELATIONS AMONG APPROACHES}

It is evident that the puzzle of absolute scale of neutrino masses connects very different branches of physics, ranging from the sub-eV scale of $0 \nu \beta \beta$ and end-point tritium decay, to the $\mathrm{ZeV}$ scale of EECRs, to the matter fluctuations of the primordial Universe. As mentioned in the introduction, the Z-burst and cosmic structure measurements are sensitive to the heavier neutrino masses, while the tritium and $0 \nu \beta \beta$ experiments are sensitive to linear combinations of masses (presumably the lighter ones) most coupled to $\nu_{e}$. The heavier and lighter masses are related by the $\delta m^{2}$ 's inferred from oscillation experiments, which in turn correlates the possible findings of the four approaches to absolute neutrino-mass determination. One way to display the correlations among the approaches is to show the overlap of their respective reaches on a mass plot. This is done in Fig. 4, where we take $0 \nu \beta \beta$ as representative of the effort to measure the lighter neutrino masses, and the Z-burst

\footnotetext{
${ }^{4}$ Were $\theta_{\text {sun }}$ truly maximal at $\pi / 4$, the dominant terms in $m_{e e}$ could cancel, leaving $m_{e e}$ beyond the reach of foreseeable $0 \nu \beta \beta$ experiments. Truly maximal mixing is not favored by fits to the data, nor by any theory.
} 
model as representative for $m_{3}$ measurement. The complementary limits from tritium decay and cosmology were already presented in the $m_{3}-m_{1}$ plane of Fig. 1.

Shown in Fig. 4 is the $0 \nu \beta \beta$-observable $m_{e e}$ predicted for each solar solution, as a function of the heaviest neutrino mass $m_{3}$, or, alternatively the Z-burst resonance energy $E_{R}=4\left(\mathrm{eV} / m_{3}\right)$ ZeV. This mass-correlated plot is possible because fixing $m_{3}$ fixes $m_{1}$ and $m_{2}$, as given by eqn. (11). According to eqn. (9), for each solar solution there results a band of allowed $m_{e e}$, reflecting the uncertainties in the relative Majorana phase difference $\phi_{12}$. The exception is the SMA solution, for which the band collapses to a unique relation between $E_{R}$ and $m_{e e}$, independent of $\phi_{12}$. For the large-mixing solutions, the allowed $m_{e e}$ varies by a factor of 4 between $\pi_{12}=0$ and $\pi_{12}=\pi$ for LMA, and a factor of $\sim 10$ for LOW-QVO.

Also shown on the Figure are the recent Heidelberg-Moscow (HM) bound on $m_{e e}$, and the expected GENIUS/EXO sensitivity to $m_{e e}$. The portion of the band for each solar model below the HM bound is the viable region. The portion of each band above the GENIUS/EXO line will be probed by these experiments. Some implications for $m_{3}$ and $E_{R}$ are evident. For example, for the SMA or $\phi_{12}=0$ solutions, $m_{3}$ is bounded from above by $0.27 \mathrm{eV}$, and $E_{R}$ is bounded from below by $15 \mathrm{ZeV}$ due to the HM exclusion; $m_{3}$ is also bounded from below by $\sqrt{\delta m_{\mathrm{atm}}^{2}} \sim 0.05 \mathrm{eV}$ and $E_{R}$ from above by $80 \mathrm{ZeV}$. As $\phi_{12}$ increases from zero to $\pi$, the $0 \nu \beta \beta$ upper bound on $m_{3}$ increases to $1 \mathrm{eV}$ and $3 \mathrm{eV}$, respectively, for the $90 \%$ C.L. LMA and LOW solutions; the $0 \nu \beta \beta$ lower bound on the Z-burst energy decreases to $4 \mathrm{ZeV}$ and $1 \mathrm{ZeV}$ for these same LMA and LOW solutions, respectively.

On may turn the correlation between $0 \nu \beta \beta$ and the Z-burst model around. As an example, if the Z-burst energy $E_{R}$ is fixed, e.g. by assuming a factor $100 \mathrm{CNB}$ density increase in the Galactic halo due to clustering, one has $m_{3} \sim 0.5 \mathrm{eV}$ and $E_{R} \sim 8 \mathrm{ZeV}$, and a resulting lower bound on $m_{e e}$ of $0.1 \mathrm{eV}$ and $0.04 \mathrm{eV}$ in the LMA and LOW models at $90 \%$ C.L., respectively. As can be seen in Fig. 4, these values of $m_{e e}$ lie within the reach of the 1 ton GENIUS and the EXO proposals. Therefore, if the GENIUS/EXO experiments fail to see $0 \nu \beta \beta$, then either neutrinos are Dirac particles, neutrino clustering in our Galactic halo is insignificant, or the Z-burst hypothesis is wrong. A more complete list of correlated inferences is now given.

If $0 \nu \beta \beta$ were to measure a value of $m_{e e}$ above $0.01 \mathrm{eV}$, then the implications for the 
Z-burst model are:

- The absolute mass $m_{3}$ and therefore the Z-burst energy $E_{R}$ will be determined with an accuracy factor of $\left(\cos 2 \theta_{\text {sun }}\right)^{-1}$, which is unity for the SMA solution but $\sim 4-10$ at present for large-mixing solutions.

- If $m_{1}$ is shown to exceed $\sim 0.05 \mathrm{eV}$, then the three neutrino masses are near-degenerate, and the absolute rate of Z-bursts is increased by three, independent of the resonant neutrino flux.

- Depending on what absolute mass scale is discovered, a factor of 100 (for $m_{3} \sim 0.5 \mathrm{eV}$ ) to $10^{3}$ (for $m_{3} \sim 1 \mathrm{eV}$ ) may be gained in the Z-burst rate due to clustering in the Galactic halo.

- The neutrino is definitely a Majorana particle, and so a factor of two more is gained in the Z-burst rate relative to the Dirac neutrino case; this is because the two active helicity states of the relativistic CNB depolarize upon cooling to populate all spin states (two active and two sterile states for Dirac neutrinos, but only the original two active states for Majoranas) [34.

If $0 \nu \beta \beta$ will not be observed with $m_{e e}$ as low as $\sim 0.01 \mathrm{eV}$, then either:

- The absolute mass $m_{3}$ is determined to be $m_{3} \simeq \sqrt{\delta m_{\mathrm{atm}}^{2}} \sim 0.5 \mathrm{eV}$; and no halo clustering and no mass-degeneracy enhance the Z-burst model.

or:

- neutrinos are Dirac particles.

Conversely, if the Z-burst model turns out to be the correct explanation of EECRs, then it is probable that neutrinos possess one or more masses in the range $m_{\nu} \sim(0.1-1) \mathrm{eV}$. Reference to Fig. 1 reveals that mass-degenerate neutrino models are then likely. Some consequences are: 
- A value of $m_{e e}>0.01 \mathrm{eV}$ results, and thus a signal of $0 \nu \beta \beta$ in the GENIUS/EXO experiments is predicted, assuming the neutrinos are Majorana particles.

- Neutrino mass is sufficiently large to affect the CMB/LSS power spectrum.

We have illustrated in some detail the correlation between $0 \nu \beta \beta$ and Z-bursts. The extension to the tritium end-point experiment and the CMB/LSS study is straightforward. At a minimum, the $0 \nu \beta \beta$ and tritium end-point experiments will cross-check each other over a significant range of $m_{1}$ (assuming of course, that neutrinos are Majoranas). And the similarity in reach of the Z-burst and the CMB/LSS approaches allows a cross-check over a significant range of $m_{3}$; in particular, independent confirmation of the existence of the CNB is available. At a maximum, each of the four experimental approaches impacts the other three, since all three $m_{j}$ 's are related by the oscillation $\delta m^{2}$ 's.

\section{DISCUSSION AND CONCLUSIONS}

The mass-squared differences inferred from oscillation interpretations of solar and atmospheric neutrino data relate the three neutrino mass-eigenvalues $m_{3}, m_{2}$, and $m_{1}$. Accordingly, only the overall mass scale is devoid of information. We have considered four approaches mixing experiment and theory which have the potential to infer a neutrino mass below $1 \mathrm{eV}$. These are $0 \nu \beta \beta$ and tritium decay end-point measurements, which in future experiments may be sensitive to the lighter masses $m_{1}$ and $m_{2}$ if they exceed 0.01 and $0.2 \mathrm{eV}$, respectively; and extreme-energy cosmic ray measurements in the context of the Z-burst model, and comparisons of cosmological measurements of CMB fluctuations and LSS distributions, which are sensitive to all neutrino masses $\gtrsim 0.1 \mathrm{eV}$. Due to the mass relations implied by the oscillation data, the findings expected from each of these four approaches is correlated with the findings expected in the other three. We have presented in some detail why and how this is so. Special emphasis was placed on $0 \nu \beta \beta$ as representative of $m_{1}, m_{2}$ measurements, and on the Z-burst model as representative of $m_{3}$ measurements. The gross correlations between $0 \nu \beta \beta$ and the Z-burst model are presented in Fig. 4. More subtle inferences are itemized in the previous section. Present constraints from the tritium decay spectrum and from CMB/LSS measurements are shown in Fig. 1. Taken together, the four 
approaches hold the potential not only to determine the absolute neutrino mass, but also to cross-check the validity of the assumptions underlying the approaches.

Of the four approaches discussed here, the Z-burst model probably contains the most speculative assumption, namely that there exists a substantial cosmic flux of neutrinos at energy $E_{R} \sim 10^{22} \mathrm{eV}$. This assumption may be checked directly [34] in a teraton neutrino detector such as the proposed EUSO/OWL/AW orbiting experiment [15. The remaining assumptions in the Z-burst model seem solid, relying only on Standard Model physics, the Standard Cosmological Model, and the existence of neutrino mass. In other words, if $F_{\nu}\left(E_{R}\right)$ is nonzero, then Z-bursts have to occur; but the rate is proportional to the completely unknown value of $F_{\nu}\left(E_{R}\right)$.

Finally, we wish to comment on what changes in this work if the neutrino masses exhibit the disfavored "inverted" spectrum. In the inverted spectrum, the two heavier states are split from one another by $\delta m_{\text {sun }}^{2}$, and separated from the remaining lighter state by $\delta m_{\text {atm }}^{2}$. With the inverted spectrum, the ordinate Fig. 1 becomes the near-degenerate masses of the two heavier states $m_{3}$ and $m_{2}$, while the abscissa becomes just the single lighter state $m_{1}$. More importantly, the $\nu_{e}$ state is mixed mainly with the two heavier states, and so the $0 \nu \beta \beta$ and tritium end-point approaches to absolute mass determination become sensitive to the heavier states, as is the case with the Z-burst and CMB/LSS approaches. In the $0 \nu \beta \beta$ section of this paper, the lighter mass $m_{1}$ in eqns. (9) and (10) is replaced with the heavier mass $m_{3}$. As a consequence, the present HM bound on $m_{e e}$ directly impacts the Z-burst model, and the potential of CMB/LSS measurements to infer a neutrino mass. For the degenerate case with $m_{1} \gg 0.05 \mathrm{eV}$, the situation is equivalent to the normal hierarchy. However, even in the strongly (inverse) hierarchical case, where $m_{1} \ll m_{3}$, it is true that $m_{e e} \gtrsim m_{3} \cos 2 \theta_{\text {sun }} \gtrsim \sqrt{\delta m_{\text {atm }}^{2}} \cos 2 \theta_{\text {sun }}$, which allows a cross-check of these approaches.

With four approaches available for determination of neutrino mass below $1 \mathrm{eV}$, there is

\footnotetext{
${ }^{5}$ It was proposed long ago [16] that spectroscopy of all neutrino masses may be done by observing the energies $\left(E_{\nu_{j}}^{R}=4\left(\mathrm{eV} / m_{j}\right) \mathrm{ZeV}\right)$ of absorption dips in the extreme-energy neutrino flux. This idea remains possible in principle, but in practice requires an even larger neutrino flux than that required for an observable rate of Z-bursts.
} 
hope that the absolute neutrino mass scale will become known. The overlap in mass-reach of the four approaches, discussed in this work, will provide an important consistency check on any positive result of any one approach.

\section{ACKNOWLEDGEMENTS}

This work was supported in part by the DOE grant no. DE-FG05-85ER40226. 


\section{REFERENCES}

[1] V. Barger, T.J. Weiler, K. Whisnant, Phys.Lett. B442 (1998) 255-258

[2] C. Weinheimer, talk at the EPS HEP 99 conference at Tampere/Finland

[3] R. Croft, W. Hu, and R. Dave, Phys. Rev. Lett. 83, 1092 (1999); M. Fukugita, G.-C. Liu, and N. Sugiyama, Phys. Rev Lett. 84, 1082 (2000); for a review, see, e.g., C-P Ma, TASI98 Lecture, Boulder, CO, ed. P. Langacker, Pub. World Sci. astro-ph9904001; J. Primack and M. Gross, in "Current Aspects of Neutrino Physics", ed. D. Caldwell, pub. Springer-Verlag, 2001 astro-ph/0007165].

[4] H.V. Klapdor-Kleingrothaus et al., to be publ. 2000 and http://www.mpihd.mpg.de/non_acc/main.htm]

[5] R. Mohapatra, in "Current Aspects of Neutrino Physics", ed. D. Caldwell, pub. Springer-Verlag, 2001 hep-ph/9910365. G. Altarelli and F.Feruglio, hep-ph/9905536, to appear in Phys. Rept.

[6] Theories with large extra dimensions can motivate small neutrino masses of either Dirac or Majorana type. Examples for Dirac masses are:

N. Arkani-Hamed, S. Dimopoulos, G. Dvali, J. March-Russell, hep-ph/9811448; K. Dienes, E. Dudas, and T. Gherghetta, Nucl. Phys. B557, 25 (1999); A. Faraggi and M. Pospelov, Phys. Lett. B458, 237 (1999); A. Das and O. Kong, Phys. Lett. B470, 149 (1999); G. Dvali, A. Yu. Smirnov, Nucl.Phys. B563 (1999) 63; R.N. Mohapatra, S. Nandi, A. Perez-Lorenzana, Phys.Lett. B466 (1999) 115; A. Ioanissian, J.W.F. Valle, hep-ph/9911349; R. Barbieri, P. Creminalli, and A. Strumia, hep-ph/0002199.

[7] The homepage for the Microwave Anisotropy Probe (MAP) is http://map.gsfc.nasa.gov/

[8] The homepage for the PLANCK Mission is http://astro.estec.esa.nl/SA-general/Projects/Planck/

[9] The Sloan Digital Sky Survey (SDSS) homepage is http://ssds.nasa.gov/ 
[10] The Two Degree Field system (2dF) homepage is http://www.ast.cam.ac.uk./AA0/2df/

[11] The homepage for the Akeno Giant Air Shower Array (AGASA) is http://www-akeno.icrr.u-tokyo.ac.jp/AGASA/

[12] The homepage for the High Resolution Fly's Eye Cosmic Ray Detector (HiRes) is http://hires.physics.utah.edu/

[13] The homepage for the Pierre Auger Project is http://www.auger.org/

[14] The homepage for the Telescope Array Project is http://www-ta.icrr.u-tokyo.ac.jp/

[15] The Extreme-Universe Space Observatory (EUSO) homepage is http://www.ifcai.pa.cnr.it/Ifcai/euso.htm]; the Orbiting Wide-angle Light-collectors (OWL) homepage is http://owl.gsfc.nasa.gov;

the AirWatch side of OWL has a homepage at http://www.ifcai.pa.cnr.it/ AirWatch/

[16] T.J. Weiler, Phys. Rev. Lett. (1982); ibid., Astrophys. J. 285, 495 (1984); E. Roulet, Phys. Rev. D47, 5247 (1993).

[17] T.J. Weiler, Astropart. Phys. 11, 303 (1999), and ibid. 12, 379E (2000) [for corrected receipt date]; D. Fargion, B. Mele and A. Salis, Astrophys. J. 517, 725 (1999).

[18] J. Beacom, R. Boyd, and A. Mezzacappa, Phys. Rev. Lett. 85, 3568 (2000).

[19] H. Minakata and H. Nunokawa, hep-ph/0010240; C. Lunardini and A. Yu. Smirnov, hep-ph/0009356.

[20] M.C. Gonzalez-Garcia, M. Maltoni, C. Peña-Garay, J.W.F. Valle, hep-ph/0009350; For earlier works see J.N. Bahcall, P.I. Krastev, A.Yu. Smirnov, Phys.Rev. D60 (1999) 093001; G.L. Fogli, E. Lisi, D. Montanino, A. Palazzo, Phys.Rev. D62 (2000) 013002; G.L. Fogli, E. Lisi, D. Montanino, A. Palazzo, Phys.Rev. D62 (2000) 113004; H. Mi- 
nakata, H. Nunokawa, hep-ph/9902460; see also M.V. Garzelli and C. Giunti, hep$\mathrm{ph} / 0012044$.

[21] M. Kachelriess, R. Tomas, and J. Valle, hep-ph/0012134, and references therein.

[22] M. Takeda et al. (AGASA Collaboration), Phys. Rev. Lett. 81, 1163 (1998) astroph/9807193]; D.J.Bird et al. (Fly's Eye Collaboration), Phys. Rev. Lett. 71, 3401 (1993), Astrophys. J. 424, 491 (1994), and ibid 441, 144 (1995); M.A. Lawrence, R.J.O.Reid and A.A. Watson (Haverah Park Collaboration), J. Phys. G 17,773 (1991), and M. Ave, J.A.Hinton, R.A.Vazquez, A.A.Watson and E. Zas, Phys. Rev. Lett. 85, 2244 (2000), and http://ast.leeds.ac.uk/haverah/hav-home.shtm]; N.N. Efimov et al. (Yakutsk Collaboration), Proc. "Astrophysical Aspect of the Most Energetic Cosmic Rays," p. 20, eds. M. Nagano and F. Takahara, World Sci., Singapore, 1991; D. Kieda et al. (HiRes Collaboration), Proc. of the 26th ICRC, Salt Lake City, Utah, 1999.

[23] Recent reviews include: P. Biermann, J. Phys. G23, 1 (1997); P. Bhattacharjee and G. Sigl, Phys. Rept. 327, 109 (2000) astro-ph/9811011]; R.D. Blandford, in "Particle Physics and the Universe", Physica Scripta, ed. L. Bergstrom et al., World Scientific astro-ph/9906026; A.V. Olinto, "David Schramm Memorial Volume" of Phys. Rept. 333, 329 (2000); X. Bertou, M. Boratov, and A. Letessier-Selvon, Int. J. Mod. Phys. A15, 2182 (2000); A. Letessier-Selvon, Lectures at "XXVIII International Meeting on Fundamental Physics", Cadiz, Spain (2000) astro-ph/0006111; M. Nagano and A.A. Watson, Rev. Mod. Phys. 72, 689 (2000); F.W. Stecker, astro-ph/0101072.

[24] Particle Data Group, Euro. Phys. J. C15 (2000) pp. 226-7.

[25] S. Yoshida, G. Sigl, and S. Lee, Phys. Rev. Lett. 81, 5505 (1998); J. Blanco-Pillado, R. Vazquez, and E. Zas, Phys. Rev. D61, 123003 (2000); G. Gelmini and A. Kusenko, Phys. Rev. Lett. 82, 5202 (1999); ibid, 84, 1378 (2000); J. Crooks, J. Dunn, and P. Frampton, astro-ph/0002089.

[26] S. Tremaine and J. Gunn, Phys. Rev. Lett. 42 (1979) 407

[27] W. Hu, D.J. Eisenstein, and M. Tegmark, Phys. Rev. Lett. 80 (1998) 5255. 
[28] R.E. Lopez, astro-ph/9909414.

[29] H.V. Klapdor-Kleingrothaus, H. Päs, A.Y. Smirnov, hep-ph/0003219, Phys. Rev D, in the press; M. Czakon, J. Gluza, J. Studnik, M. Zralek, hep-ph/0010077, and references therein.

[30] J. Hellmig, H.V. Klapdor-Kleingrothaus, Z. Phys. A 359 (1997) 351; H.V. KlapdorKleingrothaus, M. Hirsch, Z. Phys. A 359 (1997) 361; H. V. Klapdor-Kleingrothaus, L. Baudis, G. Heusser, B. Majorovits, H. Päs, hep-ph/9910205.

[31] M. Danilov et al., Phys.Lett. B480 (2000) 12.

[32] M. Apollonio et al. (CHOOZ collab.), hep-ex/9907037, Phys. Lett. B 466 (1999) 415.

[33] V. Barger, D. Marfatia, B.P. Wood, hep-ph/0011251; H. Murayama and A. Pierce, hep-ph/0012075.

[34] T.J. Weiler, in "Beyond the Desert 99, Ringberg Castle", Tegernsee, Germany, June 6-12, 1999 hep-ph/9910316. 


\section{FIGURES}

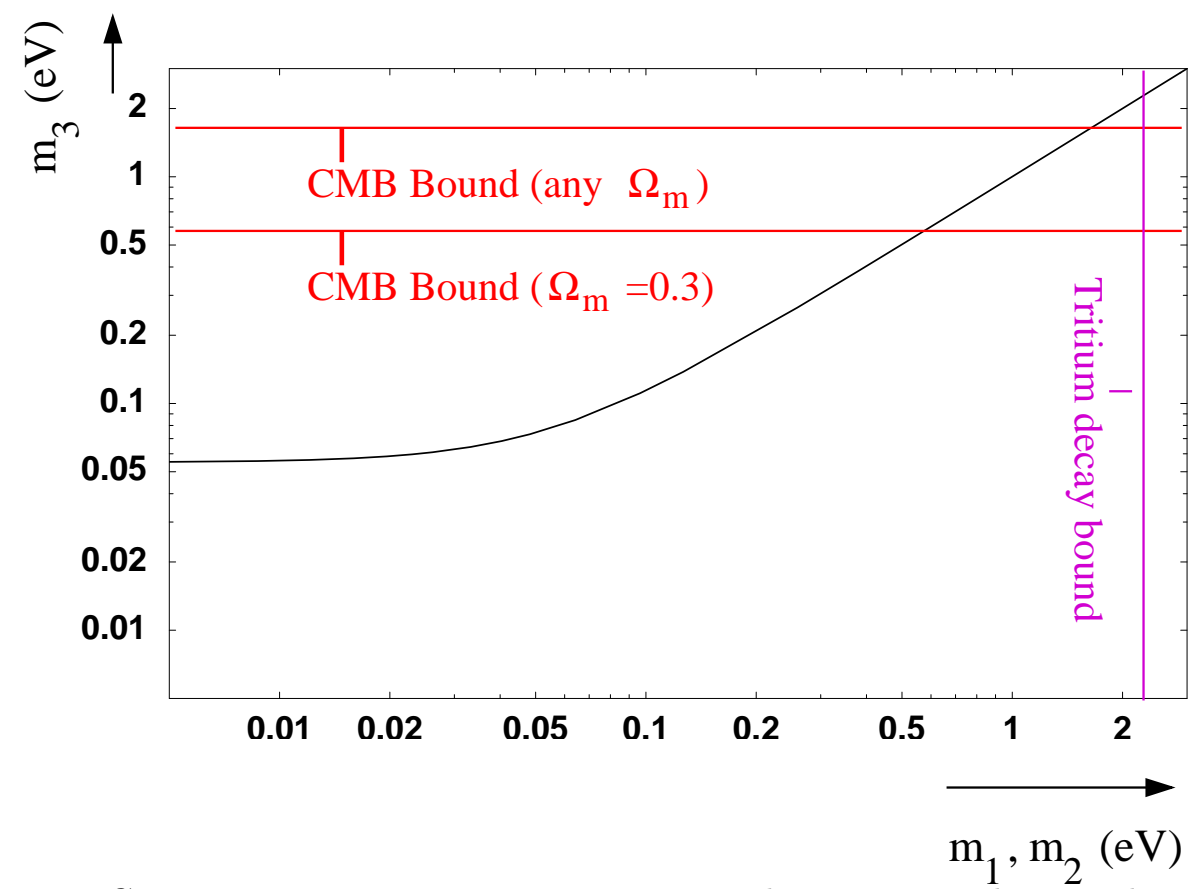

FIG. 1. Neutrino mass constraints in the $m_{1,2}-m_{3}$ plane. The curved line corresponds to allowed values according to the solar and atmospheric neutrino data. Direct mass measurements from CMB and tritium beta decay exclude the regions beyond their respective straight lines. 


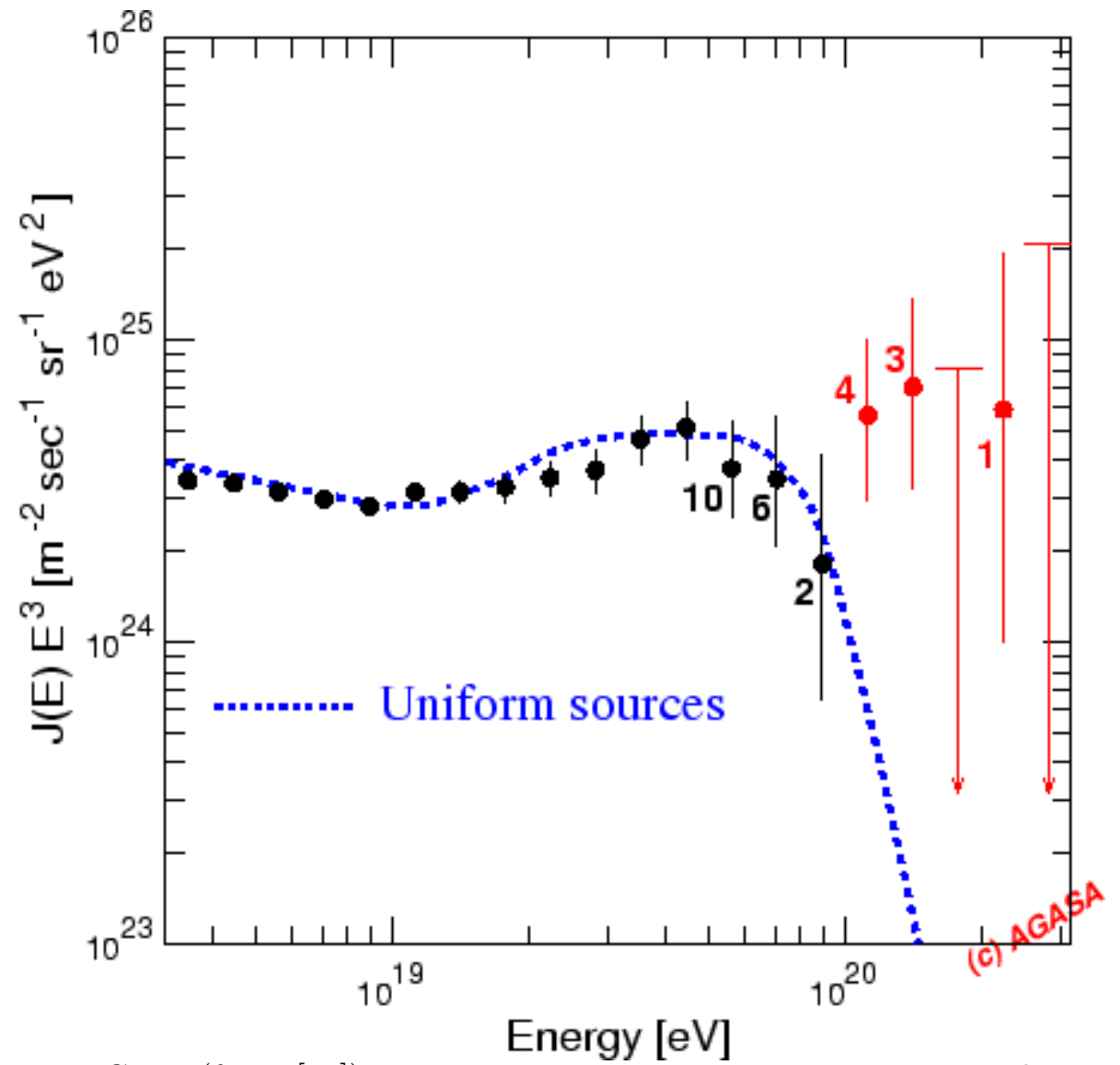

FIG. 2. (from [11]) Extreme-energy cosmic ray spectrum as observed by AGASA. Error bars correspond to $68 \%$ C.L. and the numbers count the events per energy bin. The dashed line revealing the GZK cutoff is the spectrum expected from uniformly distributed astrophysical sources. 


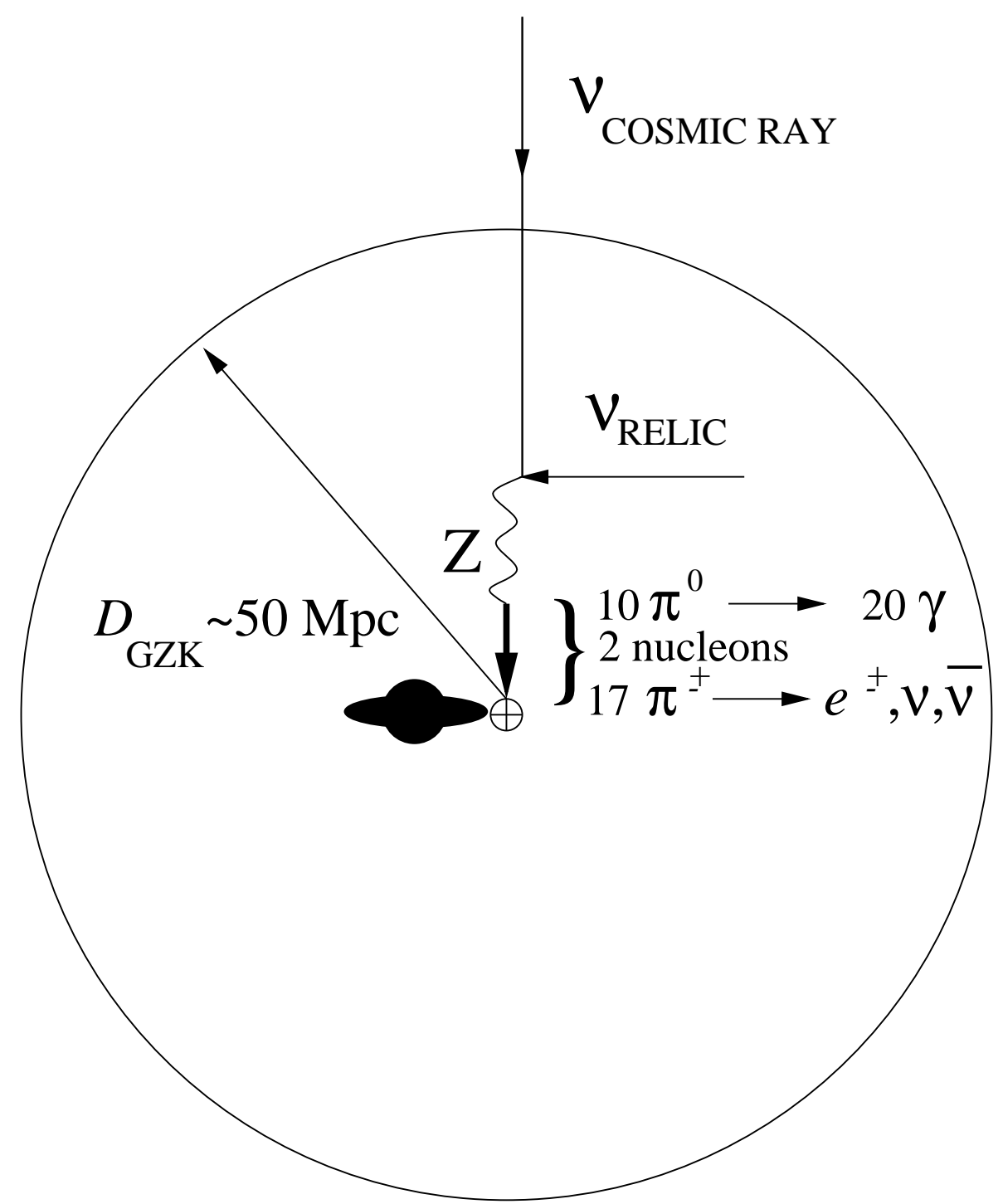

FIG. 3. Schematic diagram showing the production of a Z-burst resulting from the resonant annihilation of a cosmic ray neutrino on a relic (anti)neutrino. If the Z-burst occurs within the GZK zone ( $\sim 50$ to $100 \mathrm{Mpc})$ and is directed towards the earth, then photons and nucleons with energy above the GZK cutoff may arrive at earth and initiate super-GZK air-showers. 


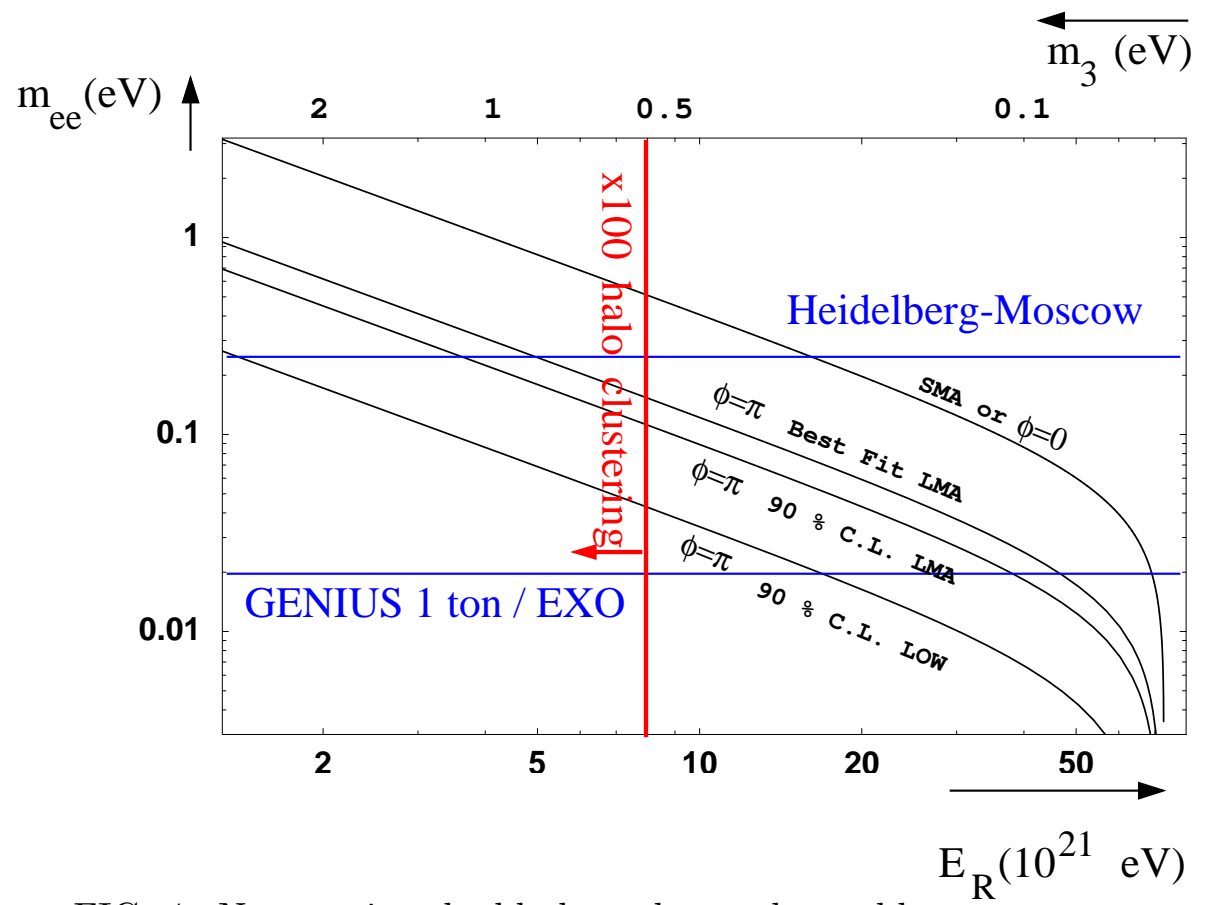

FIG. 4. No-neutrino double beta-decay observable $m_{e e}$ versus mass of the heaviest neutrino $m_{3}$, or, alternatively, the resonant Z-burst energy $E_{R}$. The curved lines show allowed regions for different solutions of the solar neutrino anomaly; from top to bottom, the case for $\phi_{12}=0$, or arbitrary $\phi_{12}$ for the SMA, and the cases $\phi_{12}=\pi$ for the LMA solution best fit, the $90 \%$ C.L. LMA solution and the $90 \%$ C.L. LOW solution. The region between the $\phi_{12}=0$ and the $\phi_{12}=\pi$ lines are allowed in the various solar solutions. The straight lines show the bound from the Heidelberg-Moscow experiment excluding the region above, the sensitivity of the 1 ton GENIUS and EXO projects, and the region where Galactic halo clustering provides a neutrino overdensity of 100 or more (moving to the left). 